\title{
Comportamento Matemático: Relações Ordinais e Inferência Transitiva em Pré-escolares ${ }^{1}$
}

\author{
Mylena Pinto Lima Ribeiro ${ }^{2}$ \\ Centro Universitário Vila Velha \\ Grauben de Assis \\ Universidade Federal do Pará \\ Sônia Regina Fiorim Enumo \\ Universidade Federal do Espírito Santo
}

\begin{abstract}
RESUMO - Um repertório matemático elementar pode ser diretamente ensinado a pré-escolares em risco de dificuldades de aprendizagem. Nesta pesquisa, examinou-se o controle do comportamento por relações ordinais em 14 crianças (idade média: 5 anos e 1 mês) com baixo rendimento escolar numa escola municipal de Vitória. Inicialmente, os estudantes foram avaliados de modo individualizado, em seguida aplicou-se um procedimento de ensino informatizado para ensinar desempenhos ordinais e avaliou-se a ocorrência de desempenhos gerativos. Todas as crianças alcançaram o critério de ensino, estabelecendo-se o controle do comportamento por relações ordinais. Na avaliação da transferência de funções de estímulo 14 crianças ordenaram novas seqüências de estímulos com o procedimento informatizado e oito crianças com o procedimento não informatizado. Conclui-se que habilidades básicas para o aprendizado da matemática podem ser diretamente ensinadas, apesar das falhas no repertório inicial da criança.
\end{abstract}

Palavras-chave: seqüências; comportamento matemático; relações ordinais; dificuldades de aprendizagem; pré-escolares.

\section{Mathematical Behavior: Ordinal Relations and Transitive Inference in Pre-scholars}

\begin{abstract}
Basic skills on mathematics can be taught directly to pre-scholars in risk of learning difficulties. This research examined the behavior control by ordinal relations in 14 children (mean age: 5 years and 1 mosnth old) with low school performance from a municipal school in Vitoria. Initially, the students were individually assessed; after that a computerized teaching procedure was applied to teach ordinal performances and then the occurrence of generative performance was assessed. All the children met the teaching criterion, thereby establishing the behavior control by ordinal relations. In the transference of stimuli function assessment, 14 children ordered new sequences of stimuli with the computerized procedure and eight children with the non-computerized procedure. We may conclude that basic skills for learning mathematics can be taught directly in spite of the failures in the initial repertoire of the child.
\end{abstract}

Key words: sequences; mathematical behavior; ordinal relations; learning difficulties; pre-scholars.

$\mathrm{Na}$ análise do comportamento matemático, considera-se que uma rede de relações entre estímulos e entre estímulos e/ou respostas estabelece as condições para a ocorrência de desempenhos verbais e de outros desempenhos cognitivos complexos, como a resolução de problemas aritméticos. A compreensão da produtividade comportamental observada nas habilidades aritméticas em humanos requer tanto a

1 Este artigo é derivado da tese de doutorado da primeira autora sob orientação dos demais autores, apresentada ao Programa de PósGraduação em Psicologia da UFES em 2004 com apoio da CAPES (Bolsa) e do CNPq (Bolsa de Iniciação Científica). Agradecimentos à Rafaela Feijó dos Santos (bolsista Iniciação Científica/CNPQ/UFES) e aos estagiários Cibele Marques Coelho de Medeiros e João Paulo Guidoni do Centro Universitário Vila Velha pelo apoio na coleta e processamento dos dados. Agradecemos ainda ao Sr. João Vicente do Nascimento pelo desenvolvimento do software (REL 3.0) de coleta de dados comportamentais.

2 Endereço: Rua Francisco Eugênio Mussiêlo, 20, apt. 203-B, Jardim da Penha, Vitória, ES, Brasil 29060-290. E-mail: mylenalima@bol. com.br investigação da formação de sequiências numéricas, que são estabelecidas por meio do treino de rotinas, quanto uma descrição das relações funcionais envolvidas na formação de classes ordinais. E mais, classes ordinais também poderiam se transferir para novos contextos? Mackay, Stromer e Serna (1998) relatam que os avanços no conhecimento acerca da formação e das funções exercidas por classes de estímulos são fundamentais no que diz respeito aos processos básicos envolvidos na generalização e na transferência de funções discriminativas. Uma estratégia bastante documentada para avaliação de desempenhos novos é o exame das relações de equivalência entre estímulos (de Rose, 1993). Classes de estímulos equivalentes emergem do estabelecimento de relações condicionais entre estímulos. A equivalência de estímulos é usualmente analisada com o auxílio do procedimento de discriminação condicional, que estabelece relações arbitrárias entre estímulos. Nas relações arbitrárias, uma resposta comum é evocada por estímulos que não compartilham semelhança física. Quando uma função de estímulo, estabelecida para um membro da classe de estímulos, trans- 
fere-se para outros membros sem qualquer ensino adicional e sem a necessidade de mediação por respostas comuns, diz-se que esses estímulos são "funcionalmente equivalentes" (Goldiamond, 1962; Sidman \& Tailby, 1982; Sidman, 1986; Sidman, 1994).

Carmo (2000) define como repertórios matemáticos os comportamentos (verbais ou não-verbais) com probabilidade de serem emitidos em contingências que envolvam números falados ou escritos, numerosidades, dígitos, problemas aritméticos e de cálculo etc. Para o ensino e avaliação do comportamento conceitual numérico pode-se recorrer ao procedimento de discriminação condicional como uma tática para estabelecer redes de relações entre estímulos numéricos. De modo resumido, nessa estratégia de ensino e avaliação tem-se, por exemplo: se um modelo é \#, então, a comparação $\{1\}$ deverá ser selecionada; se \#\# então será $\{2\}$, selecionado; se \#\#\#, então \{3\} será selecionado, e assim por diante. A ocorrência desses desempenhos mostra a formação de três classes de estímulos, cada uma consistindo de um nome ditado, uma quantidade de itens e um dígito ou numeral, ou seja, $\{$ "Um"/ \# /1 \}, \{“dois"/ \#\#/ 2\}, e \{"três"/ \#\#\#/ 3 \} (Carmo, 2000; Carmo, 2002; Carmo \& Prado, 2004; Prado, 2001).

O comportamento matemático pode ser avaliado com o auxilio do paradigma de equivalência ao se enfatizar as relações ordinais como protocolo básico. Por definição, ordenação é um tipo de responder seqüencial que ocorre na presença de um conjunto de estímulos dispostos de forma simultânea e que está sob controle de propriedades sutis de relações entre estímulos. Relações ordinais estão presentes no uso de vocábulos relacionais, como, por exemplo, no vocabulário dêitico (ver Catania, 1984/1999) e nos intraverbais, como a contagem, e na organização de frases e sentenças (Assis \& Costa, 2004; Assis, Élleres \& Sampaio, 2006; Lazar \& Kotlarchyk, 1986; Mackay, Kotlarchyk \& Stromer, 1997; Skinner, 1957/1992; Stromer \& Mackay, 1990).

As regularidades encontradas na pesquisa sobre relações ordinais sugerem a possibilidade de reavaliação da função do encadeamento de estímulos (ver Whaley \& Malott, 1971/1981) no que concerne à aprendizagem serial e ao comportamento verbal. Diferentemente do protocolo tradicional para o exame das relações de equivalência, no exame do comportamento controlado por relações ordinais enfatizam-se as contingências de reforçamento de três termos. Pergunta-se como o responder ordinal pode ser controlado por eventos fisicamente dessemelhantes, que aparecem em uma sucessão temporal, sem que tais estímulos tenham sido anteriormente relacionados. Em outras palavras, avalia-se se novos desempenhos ordinais estariam baseados na substituição de estímulos, considerada uma medida de inferência transitiva.

Entre as táticas utilizadas para o estudo das relações ordinais tem-se o ensino da ordenação via encadeamento de estímulos (chaining procedures) e por justaposição de estímulos (overlapping) (Green, Stromer \& Mackay, 1993; Holcomb, Stromer \& Mackay, 1997). No ensino por encadeamento, a tarefa do aprendiz é tocar cada estímulo em uma ordem específica, sem importar sua localização espacial. Por exemplo, se o arranjo disponível de estímulos é \#\#\#, \#, e \#\#, logo que o sujeito os toque na ordem \#>\#\#>\#\#\# seu desempenho é reforçado.
Um estudo clássico na literatura foi desenvolvimento por Lazar (1977), que sugeriu o exame da transferência de funções de estímulos envolvendo a noção de posição ordinal. Posteriormente, procurou-se verificar os efeitos de variações de procedimentos de treino sobre os desempenhos ordinais e sobre a formação de novas seqüências de estímulo (Assis \& Costa, 2004; Assis \& Sampaio, 2003; Galy, Camps \& Melan, 2003; Green \& cols., 1993; Lima \& Assis, 2003; Maydak, Stromer, Mackay \& Stoddard, 1995; Sampaio \& Assis, 2005; Sigurdardottir, Green \& Saunders, 1990; Stromer \& Mackay, 1993).

A análise das relações entre estímulos pode destacar estímulos de uma seqüência e estímulos componentes de seqüências ensinadas de modo independente umas das outras. Para isto, Green e cols. (1993) propõem a realização de testes comportamentais para o exame das propriedades de uma relação ordinal, tal como apresentado na matemática por Stevens (1951). A análise descritiva de relações estímulo-estímulo que ocorrem nas seqüências de estímulos evidencia as relações ordinais. A propriedade irreflexividade pressupõe que uma relação ordinal nunca é reflexiva, logo, é verdadeira a proposição $\{\mathrm{A} 1>\mathrm{A} 1\}$. A propriedade assimetria informa que uma relação ordinal é sempre unidirecional, assim, se $\{\mathrm{A} 2>\mathrm{A} 3\}$ está correto, então, $\{\mathrm{A} 3>\mathrm{A} 2\}$ está incorreto. Numa relação ordinal, a transitividade requer, após o ensino de $\{\mathrm{A} 2>\mathrm{A} 3\}$ e $\{\mathrm{A} 3>\mathrm{A} 4\}$, que novos desempenhos de ordenação $\{\mathrm{A} 2>\mathrm{A} 4\}$ ocorram sem ensino adicional. Note-se que apenas pares de estímulos não-adjacentes em séries previamente ensinadas permitem a inferência de transitividade. E, finalmente, a propriedade conectividade informa que numa relação ordinal todos os pares de estímulos estão correlacionados. Assim, se $\{\mathrm{A} 1>\mathrm{A} 2>\mathrm{A} 3\}$, então $\{\mathrm{A} 1>\mathrm{A} 2\},\{\mathrm{A} 1>\mathrm{A} 3\}$ e $\{\mathrm{A} 2>\mathrm{A} 3\}$ é verdadeiro. Logo, existe conectividade se todos os pares possíveis de uma seqüência e de seqüências diferentes podem ser formados, tanto pares adjacentes como os pares não-adjacentes.

A relação de controle entre estímulos não-adjacentes de uma seqüência nova favorece a análise da inferência transitiva e exclui uma explicação pautada na noção de encadeamento, na qual a função de "elo", usualmente atribuída a um estímulo discriminativo, não está evidenciada. A transitividade, no entanto, não pode ser isolada se o procedimento de ensino que precedeu a prova incluiu a apresentação de todos os estímulos que formam a sequiência (por exemplo, A1>A2>A3>A4>A5). Por isso, o ensino de duas ou mais sequiências independentes, seguido por provas com sequiências mistas, é considerado mais apropriado para o exame do potencial transitivo. Assim, a substituição de estímulos que ocuparam as mesmas posições ordinais nas sequiências ensinadas de modo independente e que resultam na ordenação de sequiências novas é incompatível com a noção de encadeamento clássico.

Em resumo, o protocolo básico para o exame de desempenhos ordinais produtivos segue os passos: a) ensino independente de duas ou mais seqüências; b) avaliação das propriedades das relações ordinais. Desse modo, pode-se sugerir que o comportamento matemático requer o estabelecimento de uma matriz relacional que inclui diferentes tipos de relações entre 
estímulos que podem ser descritas nos termos das relações de equivalência e, adicionalmente, das relações ordinais.

Neste estudo buscou-se examinar as relações envolvidas na formação de classes ordinais e transferência de funções de estímulos em pré-escolares. Foi enfatizado o exame das propriedades que caracterizam desempenhos ordinais através dos seguintes passos: a) descrição do repertório de entrada de crianças pré-escolares; b) ensino de desempenhos ordinais com um conjunto de estímulos numéricos; c) avaliação da formação de classes ordinais e d) avaliação da expansão de classes ordinais (transferência de funções de estímulo).

\section{Método}

\section{Participantes}

Participaram da pesquisa 14 pré-escolares (idade média: 5 anos e 1 mês), selecionados entre os alunos de uma pré-escola do município de Vitória-ES, 7 meninas e 7 meninos. Solicitou-se às professoras que indicassem crianças que, em sua opinião, apresentassem dificuldades na consecução de tarefas escolares. Foram, assim, indicadas 24 crianças. Este número foi reduzido a 14 crianças por meio da exclusão dos alunos que apresentaram $100 \%$ de respostas corretas no pré-teste. A pesquisa foi conduzida na própria escola. A sala de coleta de dados constituía um ambiente agradável, com isolamento acústico, sistema de ventilação e iluminação adequada. O mobiliário incluía uma mesa escolar infantil e três cadeiras apropriadas à estatura da criança. Os pais ou responsáveis foram informados sobre a pesquisa e assinaram um termo de compromisso conforme exigência do Conselho Nacional de Saúde (Resolução 196/96).

\section{Instrumentos}

Foram utilizados na coleta de dados um computador portátil Logger (Pentium III 650 Mhz 128 Mb RAM HD $6 \mathrm{~Gb}$, tela 14 polegadas). Para a fase de ensino e avaliação de relações ordinais em contexto informatizado foi utilizado o sistema computadorizado para o ensino de leitura e de relações condicionais e seqüenciais - REL 3.0. (Nascimento, Assis \& Baptista, 2002). Para a avaliação da transferência de funções de estímulos foi utilizado o jogo pedagógico "Quantos tem?" (Mindim Brinquedos e Adaptações, Belo Horizonte, MG).

\section{Estímulos}

Os estímulos nas fases de ensino e de avaliação do procedimento informatizado foram: a) dois conjuntos de figuras familiares digitalizadas agrupadas em numerosidades de um a cinco estímulos; b) um conjunto de dígitos de um a cinco; c) nomes de dígitos de um a cinco (Figura 1).

Os estímulos usados na fase de avaliação de transferência de funções de estímulo para o contexto não-informatizado foram: a) um conjunto de figuras familiares agrupadas em numerosidades que formavam conjuntos de um a cinco estímulos e que constituíam um jogo pedagógico; b) peças

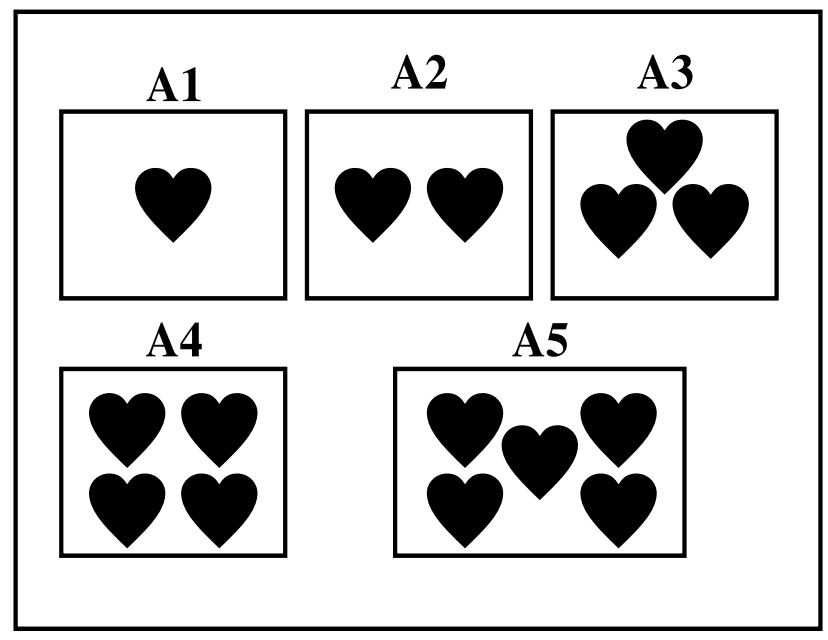

Figura 1. Estímulos experimentais na fase de intervenção com procedimento informatizado: Conjunto A (numerosidades); Conjunto B (numerosidades); Conjunto C (dígitos); Conjunto D (palavras).

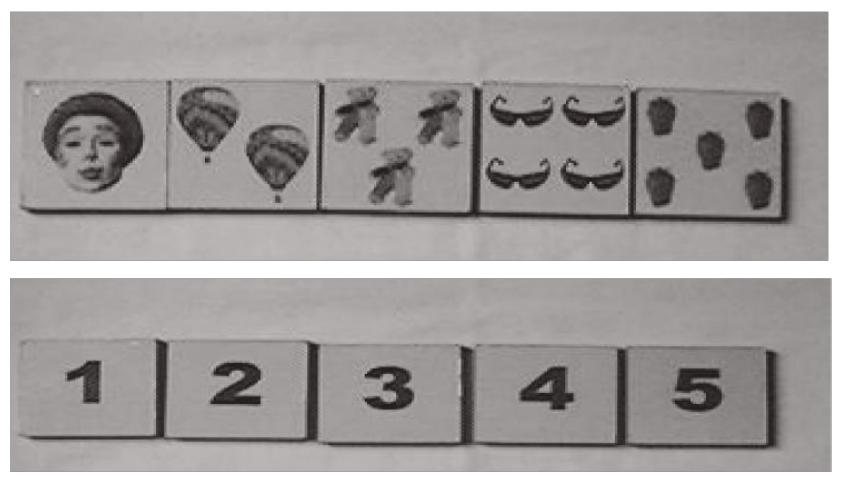

Figura 2. Estímulos experimentais utilizados na avaliação de transferência de funções de estímulo: Conjunto E (numerosidades) e F (dígitos).

de um jogo pedagógico constituídas por dígitos do numeral 1 ao numeral 5 (Figura 2).

\section{Procedimento}

As sessões individuais ocorreram em dois ou três encontros semanais, com duração média de 20 minutos. Na primeira sessão, o pré-teste foi conduzido com o objetivo de caracterizar o repertório inicial das crianças em relação às habilidades envolvidas no comportamento conceitual numérico. O procedimento consistia na apresentação de estímulos visuais (cartões impressos) com dígitos e numerosidades. As crianças eram avaliadas quanto às habilidades de nomeação de dígitos, correspondência numerosidade-dígito e desempenho de ordenação de numerosidades e de dígitos.

$\mathrm{O}$ experimento foi dividido em duas fases: I) Fase de ensino e avaliação das propriedades de relações ordinais e II) Fase de avaliação de transferência de funções de estímulo. Durante o procedimento, a criança sentava em frente ao microcomputador portátil, ficando a pesquisadora a seu lado, monitorando a sessão experimental.

Primeiramente, foi ensinado às crianças o desempenho de ordenação por meio de encadeamento. Pediu-se a ordenação 
de quatro seqüências diferentes com os seguintes conjuntos de estímulos: corações (SA), estrelas (SB), dígitos (SC) e palavras (SD). Cada seqüência completa possuía cinco estímulos componentes. Inicialmente, a tarefa do participante era selecionar o estímulo que ocupava a primeira posição em uma série. Um procedimento de cinco passos serviu para acrescentar um a um quatro estímulos restantes, por exemplo: 1) $\mathrm{A} 1$; 2) $\mathrm{A} 1>\mathrm{A} 2$; 3) $\mathrm{A} 1>\mathrm{A} 2>\mathrm{A} 3$; 4) $\mathrm{A} 1>\mathrm{A} 2>\mathrm{A} 3>\mathrm{A} 4$; 5) $\mathrm{A} 1>\mathrm{A} 2>\mathrm{A} 3>\mathrm{A} 4>\mathrm{A} 5$.

Uma tentativa do bloco de ensino iniciava-se com os estímulos dispostos na parte inferior da tela do computador, designada "área de escolha". Neste momento, era fornecida a instrução: “Toque a figura”. Após a emissão da resposta de escolha pelo participante, a pesquisadora clicava no mouse e o estímulo deslocava-se da parte inferior para a parte superior da tela, chamada "área de construção". Uma nova tentativa iniciava-se com a apresentação randomizada do primeiro estímulo e a introdução de um novo estímulo. A criança deveria apontar o primeiro estímulo e, em seguida, o novo estímulo, que passava a ocupar a segunda posição na sequiência, e assim sucessivamente. Em resumo, o participante deveria apontar primeiro $\{\mathrm{A} 1\}$, e depois $\{\mathrm{A} 2\}$, em seguida $\{$ A3 $\}$, então $\{$ A4 $\}$ e finalmente $\{$ A5 $\}$. Além disto, deveria repetir a sequiência de escolhas mais duas vezes. A instrução foi retirada gradualmente. $\mathrm{O}$ critério para avançar no programa de treino sem erro era responder corretamente em $90 \%$ das tentativas do bloco de ensino.

Conseqüências diferenciais eram apresentadas após a escolha correta da seqüência completa dos estímulos. Após a emissão de uma resposta correta, o computador apresentava a figura de um palhaço e o som de aplausos e, além disto, a pesquisadora entregava uma ficha plástica ao participante. Outras conseqüências verbais ("Muito bom!", "Você acertou!", “Você é o campeão!") também foram apresentadas. As fichas recebidas ao longo da sessão poderiam ser trocadas por brindes relacionados com o contexto escolar, como colas coloridas, figuras adesivas, lápis de cor, borrachas etc. A seleção de estímulos na ordem incorreta era seguida por um escurecimento da tela, por um intervalo entre tentativas com duração de 1s e pela reapresentação da tentativa com os mesmos estímulos, ocupando novas posições na área de escolha. Nas tentativas iniciais, a pesquisadora repetia a seguinte instrução: "O palhacinho não apareceu. Quando você fizer certo ele irá aparecer" $e$ "Vamos tentar novamente".

A avaliação de relações ordinais previa a realização das seguintes provas: a) formação de seqüência com cinco estímulos; b) formação de seqüência com pares não-adjacentes e c) formação de sequiências mistas.

O objetivo da prova de ordenação com cinco estímulos foi avaliar se os estímulos que compunham a seqüência estariam relacionados entre si, como previsto pelas propriedades irreflexividade e assimetria, ocupando cada um dos estímulos uma posição ordinal específica (se A1, segue-se A2). A prova consistia na apresentação simultânea dos cinco estímulos componentes da seqüência previamente ensinada. Nenhuma conseqüência diferencial era fornecida, a instrução fornecida à criança era: "Agora o palhaço não vai mais aparecer, mas você receberá seu brinde no final da sessão”.

A prova de ordenação de estímulos com pares nãoadjacentes buscou avaliar o potencial transitivo da tarefa de ordenação. Uma tentativa da prova começava com a apresentação de 2 estímulos pertencentes à seqüência de cinco estímulos, sendo que a tarefa do participante era escolher os estímulos na ordem correta (Se A2 e A4 então: A2 > A4). Todas as combinações possíveis com pares que ocupavam posições não-adjacentes dentro da seqüência ensinada foram avaliadas. A pesquisadora fornecia a instrução "Olhe para as figuras. Agora, você terá que tocar uma figura de cada vez. Só que, desta vez, não haverá som nem aparecerá uma figura em movimento, mas você ganhará suas fichas no final da sessão”.

A prova de múltipla substituição de estímulos ou prova mista teve por objetivo avaliar se os estímulos que formavam diferentes sequiências, que tinham sido ensinadas de modo independente, eram mutuamente substituíveis. Tratou-se de examinar se os estímulos que ocupavam a mesma posição ordinal, posições adjacentes e não-adjacentes, estariam relacionadas entre si, o que evidenciaria a propriedade de conectividade. Durante a prova de múltipla substituição de estímulos, os estímulos apareciam misturados na área de escolha e solicitava-se à criança que organizasse os estímulos, produzindo, por exemplo: $\{\mathrm{A} 1>\mathrm{B} 2>\mathrm{A} 3>\mathrm{B} 4>\mathrm{A} 5\}$, escolhendo entre os estímulos que apareciam na tela, até que nenhum estímulo restasse. Dois conjuntos de estímulo diferentes foram apresentados, sendo um conjunto por vez. Para cada conjunto era necessária a ordenação em duas tentativas. $\mathrm{O}$ critério de acerto era de $100 \%$. As tentativas não eram seguidas por conseqüências diferenciais explícitas.

Finalmente, examinou-se a transferência de funções de estímulo para um novo contexto, por meio de provas de formação de seqüências e de emparelhamento por identidade. Foram requeridos: a) ordenação de dígitos impressos, formando uma seqüência com cinco estímulos; b) a ordenação de conjuntos de dígitos impressos formando seqüências com pares de estímulos não-adjacentes e c) desempenhos de escolha de acordo com o modelo (numerosidades-dígitos impressos).

Na prova de transferência de funções de estímulos buscou-se avaliar o desempenho dos participantes em tarefas que exigiam as habilidades ensinadas diretamente pelo programa de ensino. Cada tentativa de ordenação era anotada como um acerto ou um erro no protocolo de registro da sessão experimental. No final, a freqüência de respostas corretas foi transformada em um percentual de acertos na prova. Para avaliar a formação de seqüências com cinco estímulos a pesquisadora dispunha os estímulos de cada conjunto, um conjunto por vez, misturados sobre uma mesa e dizia: "Faça uma fila com essas figuras”. Uma tentativa correta requeria a ordenação dos estímulos, dígitos de 1 a 5 ou numerosidades com conjuntos de um a cinco itens. Avaliou-se, também, o desempenho ordinal com pares de estímulos não-adjacentes. A pesquisadora apresentava dois estímulos que ocupavam posições não-adjacentes nas seqüências ensinadas e dizia "E agora, como fica a fila?". Por último, foram requeridos desempenhos de escolha de acordo com o modelo. Sobre uma mesa, a experimentadora apresentava um arranjo composto por um estímulo modelo (dígito) e três estímulos de comparação (numerosidades). A pesquisadora organizava estímulos sobre a mesa e, apontando para o estímulo-modelo, dizia: "Aponte aquele que combina com este aqui”. 


\section{Resultados}

Os resultados do pré-teste indicam que entre as 14 crianças da amostra, oito crianças $(0,57)$ não eram capazes de nomear os dígitos corretamente; três crianças $(0,21)$ eram capazes de nomear corretamente os dígitos de 1 a 3 , e três crianças $(0,21)$ eram capazes de nomear corretamente os dígitos de 1 a 5 . Considerando a relação numerosidade - dígito impresso, os resultados mostram que oito crianças $(0,57)$ não eram capazes de relacionar quantidade de itens ao dígito correspondente e seis crianças $(0,42)$ cometeram erros na tarefa. Nenhuma criança era capaz de ordenar corretamente uma seqüência com cinco estímulos, formada por dígitos impressos ou numerosidades.

Todos os participantes atingiram o critério de $100 \%$ de acertos na fase de ensino. Considerando os quatro passos para o ensino da seqüência A, 0,92 das crianças foram reexpostas ao Passo 1; enquanto 0,71 precisaram de reexposição ao Passo 2; nove crianças $(0,64)$ precisaram repetir o Passo 3 e cinco crianças $(0,35)$ precisaram de reexposição ao Passo 4. Vê-se que o desempenho, ao longo da fase de ensino da seqüência $\mathrm{A}$, foi gradualmente se tornando mais efetivo. $\mathrm{Na}$ fase de ensino da sequiência $\mathrm{B}$ os dados indicam que uma criança $(0,07)$ precisou ser reexposta ao Passo 1 , três crianças $(0,21)$ precisaram repetir o Passo 3 e oito crianças $(0,57)$ precisaram repetir o Passo 4.

$\mathrm{Na}$ fase de ensino da seqüência $\mathrm{C}$, uma criança $(0,07)$ precisou repetir o Passo 3 e cinco crianças $(0,35)$ precisaram ser reexpostas ao Passo 4. Em relação à seqüência $\mathrm{D}$, duas crianças $(0,14)$ precisaram de reexposição ao Passo 1 , duas crianças $(0,14)$ para o Passo 2 , três crianças $(0,21)$ precisaram de reexposição ao Passo 3 e 10 crianças $(0,71)$ precisaram de reexposição ao Passo 4.

Estes dados informam que o procedimento de ensino garantiu a instalação de um responder consistente. No geral, a ordenação de palavras impressas foi realizada com relativa dificuldade pelas crianças, mesmo assim, o número de reexposições ficou abaixo daquele encontrado no ensino da seqüência $\mathrm{A}$, sugerindo que a história de treino foi relevante e que, mesmo para o conjunto de estímulos complexos, o procedimento de ensino foi eficaz para instalar o desempenho de ordenação.

Após o procedimento de ensino de desempenhos de ordenação as propriedades de relações ordinais foram avaliadas. Os resultados nas provas de ordenação com cinco estímulos mostram que sete crianças $(0,5)$ apresentaram desempenho $100 \%$ correto na prova para avaliação das seqüências com cinco estímulos. Quatro crianças $(0,28)$ apresentaram erros na ordenação da Sequiência A. Três crianças $(0,21)$ apresentaram erros na ordenação da Seqüência B e três crianças $(0,21)$ apresentaram erros na ordenação da Sequiência D. Todas as crianças produziram corretamente a Sequiência C. Os erros cometidos pelas crianças implicavam em inversões na ordem em que a série deveria ser ordenada, isto é, em ordem crescente, previamente estabelecida. Esses resultados sugerem que, para a maioria dos participantes, o desempenho de ordenação estava baseado nas propriedades assimétricas das relações ordinais (Figura 3).

A prova de ordenação de pares não-adjacentes visou examinar a propriedade transitividade. Dez crianças $(0,71)$

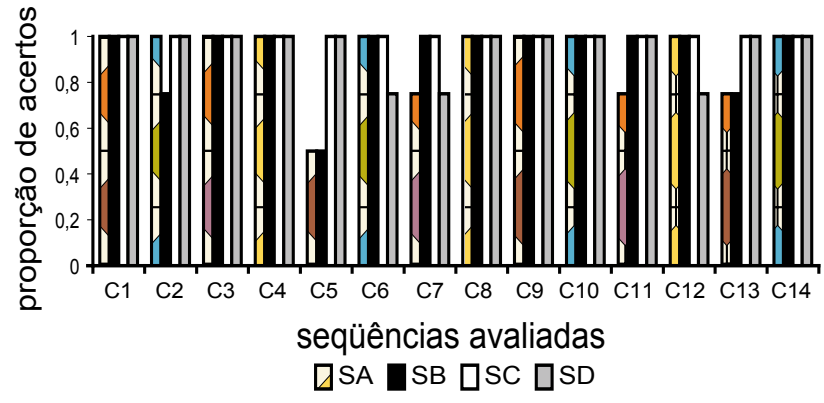

Figura 3. Proporção de acertos, por criança, na prova de formação de seqüências com cinco estímulos após procedimento de ensino $(N=14)$.

obtiveram $100 \%$ de acertos em todas as tentativas, sendo que esses participantes responderam corretamente nas seis tentativas de ordenação de pares não-adjacentes com estímulos provenientes das seqüências ensinadas previamente. Quatro crianças $(0,28)$ cometeram um erro em apenas uma das tentativas (Figura 4).

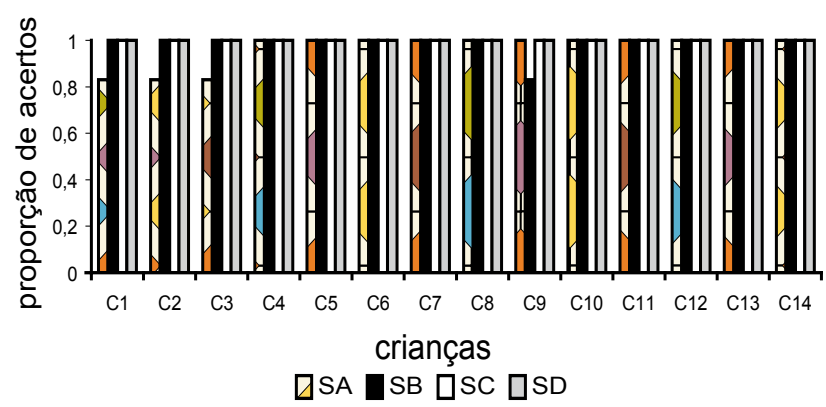

Figura 4. Proporção de acertos, por criança, na prova de ordenação com pares de estímulos não-adjacentes $(N=14)$.

A prova mista avaliou se os estímulos que ocupavam a mesma posição ordinal em sequiências ensinadas separadamente eram intercambiáveis. Foram avaliados dois conjuntos de estímulos, que misturavam estímulos provenientes das sequiências A e B. Os resultados mostram que nove crianças $(0,64)$ apresentaram um desempenho $100 \%$ correto na ordenação da primeira prova mista; quatro crianças $(0,28)$ acertaram $75 \%$, cometendo pelo menos uma inversão na ordenação da seqüência; uma criança $(0,07)$ acertou $50 \%$ e uma criança $(0,07)$ errou a tentativa. Para a segunda seqüência mista avaliada, os resultados informam que 10 crianças $(0,71)$ acertaram $100 \% ;(0,07)$ acertou $75 \%$; uma criança $(0,07)$ acertou $50 \%$ das tentativas e uma criança $(0,07)$ acertou apenas $25 \%$ das tentativas. Os desempenhos corretos acima de $71 \%$ informam que os participantes apresentaram ordenação de estímulos de acordo com a exigência da prova, respondendo acima do nível de chance. Desse modo, pode-se verificar que nas situações em que foi requerida a substituição de um estímulo por um estímulo proveniente de uma seqüência diferente, as crianças conseguiram realizar a tarefa com eficácia. Estes dados confirmam a propriedade conectividade (Figura 5). 


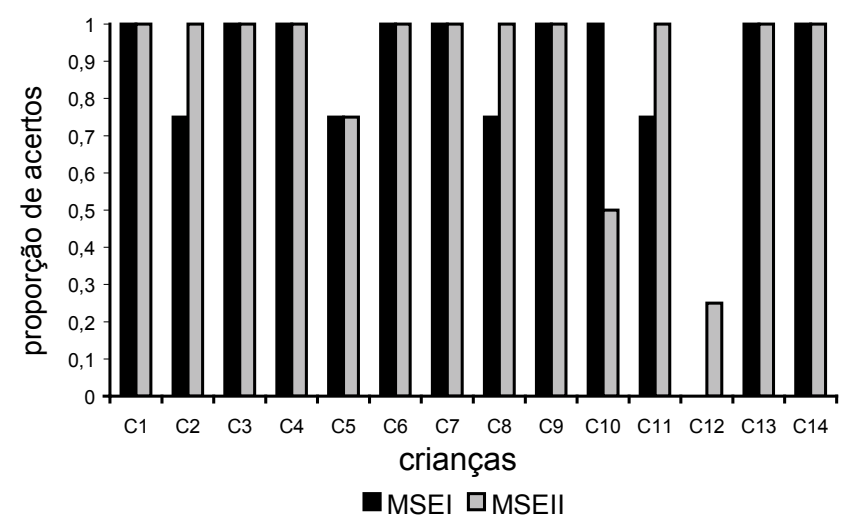

Figura 5. Proporção de acertos, por criança, nas duas provas de sequiências mistas $(N=14)$.

Os resultados obtidos no contexto não-informatizado informam desempenhos consistentes para a maioria dos participantes $(N=13)$. Oito crianças $(0,6)$ apresentaram desempenho $100 \%$ correto na prova de ordenação de seqüência com cinco estímulos; sete crianças $(0,53)$ na prova de ordenação de pares não-adjacentes e sete crianças $(0,53)$ na prova de escolha de acordo com o modelo. A análise dos desempenhos mostrou que três crianças $(0,23)$ acertaram $80 \%$ das tentativas na prova de escolha de acordo com o modelo.

Entre as crianças que tiveram maiores dificuldades, com desempenho inferior a $50 \%$ de acerto, estavam cinco crianças $(0,38)$ na prova de ordenação de seqüência com cinco estímulos, cinco crianças $(0,5)$ na prova de ordenação de pares não adjacentes e três crianças $(0,22)$ na prova de escolha de acordo com o modelo. Uma criança não acertou qualquer das tentativas e uma criança não foi submetida à prova (Figura 6). O desempenho dos participantes na ordenação de dígitos impressos com as peças do jogo pedagógico informou que houve transferência de funções de estímulo de um contexto de tarefa de ordenação informatizada para um contexto nãoinfomatizado para oito participantes.

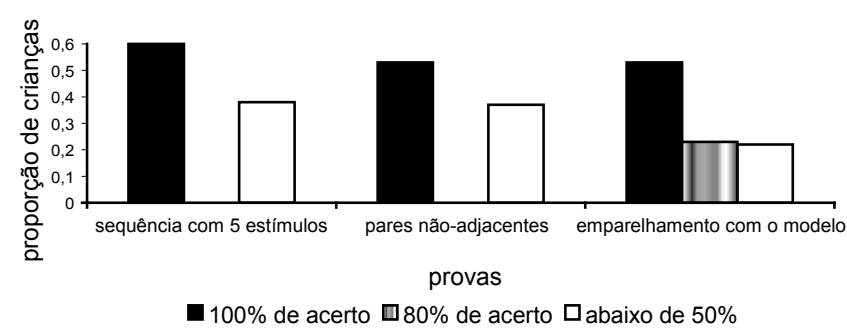

Figura 6. Proporção de crianças segundo as porcentagens de acerto nas provas do procedimento não-informatizado $(N=13)$.

\section{Discussão}

A aprendizagem da matemática relaciona-se estreitamente com a aquisição da linguagem, entre outros comportamentos complexos. Tais processos podem ser compreendidos a partir de uma perspectiva comportamental e, desse modo, sua evolução deve ser examinada dentro dos critérios que remetem à análise dos princípios básicos do comportamento humano (Skinner, 1968/1972; Hübner \& Marinoti, 2002). Considerando o aprender como um processo que evolui a partir das interações organismo-ambiente, a avaliação da novidade comportamental é crítica para a compreensão da cognição e, por isso, os critérios para a medida da inferência transitiva precisam estar bem definidos.

Numa perspectiva contemporânea das dificuldades de aprendizagem, a identificação precoce dos fatores de risco para o atraso escolar é um subsídio importante para a prevenção (Dias, Enumo, Turini \& Ferrão, 2003; Enumo \& cols., 2002). Nesta perspectiva, independentemente da presença ou da ausência de comportamentos considerados como pré-requisitos, qualquer criança exposta a um programa de ensino eficaz pode ser beneficiada, desde que seu repertório seja previamente avaliado e que as contingências de ensino sejam programadas a partir de suas necessidades. Assim, para a avaliação do comportamento conceitual numérico e de suas falhas é relevante o estabelecimento das relações funcionais entre o repertório de entrada da criança e sua historia de vida e, em especial, a história de escolarização.

Especificamente neste estudo os resultados mostram que, no pré-teste e na fase inicial do procedimento de ensino, o desempenho das crianças foi bastante heterogêneo. Gradativamente, esta dificuldade diminuiu e todos os participantes alcançaram $100 \%$ de acertos no final da fase de ensino de cada seqüência. Por outro lado, há que se considerar que no ensino de desempenhos ordinais via encadeamento houve exposição prévia a todos os estímulos que compõem a seqüência, o que pode ter interferido nos resultados da transitividade. A obtenção do critério de acerto e de elevados porcentuais de acerto nas provas de relações emergentes não constitui a única medida a ser considerada para a ocorrência das topografias de controle programadas (Dube \& McIlvane, 1996; McIlvane, Serna, Dube \& Stromer, 2000). Nesse sentido, questiona-se se os resultados positivos encontrados nas provas com pares não-adjacentes estariam relacionadas ao efeito da exposição prévia à sequiência com cinco estímulos ou se mostram desempenhos emergentes (Green \& cols. 1993; Stromer \& Mackay, 1993).

Assim, a descrição das relações funcionais entre o procedimento de avaliação e a emergência da inferência transitiva parece requerer uma verificação mais sistemática. Esse exame pode ser realizado em novos estudos por meio da introdução de novas variáveis de controle de estímulo, entre as quais: introdução de tarefas com substituição entre os estímulos de modo mais extensivo; treino por justaposição (overlapping two-stimuli), variação da disposição dos estímulos no conjunto de numerosidades; introdução de estímulos auditivos; avaliação de desempenhos de nomeação.

Os resultados negativos obtidos por três crianças mostram que essas requerem atenção individualizada, sem o que persistirá o risco para o atraso escolar em Matemática. Os dados obtidos na fase de transferência de funções de estímulo em tarefa não-informatizada mostram que, ao menos para as relações ensinadas, houve uma mudança positiva do repertório de habilidades numéricas para a maioria das crianças. Deste modo, a principal contribuição deste estudo 
relaciona-se com a exploração do potencial gerativo das relações ordinais.

O ensino de habilidades básicas em matemática deve enfatizar o responder ordinal como um ramo da rede de relações entre estímulos e entre estímulos e respostas envolvidas no comportamento conceitual matemático. A avaliação do repertório inicial explicita os déficits de repertório e, conseqüentemente, as necessidades individuais que precisam ser atendidas pela programação do ensino. Assim, o uso de tecnologia comportamental pode contribuir para a prevenção das dificuldades de aprendizagem na medida em que estabelece as condições para a ocorrência de desempenhos gerativos, modificando positivamente o repertório de entrada desses indivíduos na etapa inicial da escolarização.

\section{Referências}

Assis, G. J. A. \& Costa, L. C. A. (2004). Emergência de relações ordinais em crianças. Interação em Psicologia, 8(2), 199-216.

Assis, G. J. A. \& Sampaio, M. E. C. (2003). Efeitos de dois procedimentos de ensino na formação de classes seqüenciais. Interação em Psicologia, 7(2), 53-62.

Assis, G. J. A., Élleres, C. F. \& Sampaio, M. E. C. (2006). Emergência de relações sintáticas em pré-escolares. Interação em Psicologia, 10(1), 19-29.

Carmo, J. S. (2000). O conceito de número como rede de ligações. Em R. R. Kerbauy (Org.), Sobre comportamento e cogniçãovol. 5: conceitos, pesquisa e aplicação, a ênfase no ensinar, na emoção e no questionamento clínico (pp. 97-113). Santo André: SET.

Carmo, J. S. (2002). Comportamento conceitual numérico: Um modelo de rede de relações equivalentes. Tese de Doutorado, Universidade Federal de São Carlos, São Carlos.

Carmo, J. S. \& Prado, P. S. T. (2004). Fundamentos do Comportamento Matemático: a importância dos pré-requisitos. Em M. M. Hübner \& M. Marinotti (Orgs.), Análise do comportamento para a educação: contribuições recentes (pp. 137-157). Santo André: ESEtec.

Catania, A. C. (1999). Aprendizagem: Comportamento, linguagem e cognição. (Deisy de Souza, Trad.) Porto Alegre: Artmed. (Trabalho original publicado em 1984)

De Rose, J. C. (1993). Classes de estímulos: Implicações para uma análise comportamental da cognição. Psicologia: Teoria e Pesquisa, 9(2), 283-303.

Dias, T. L., Enumo, S. R. F., Turini, F. A. \& Ferrão, E. S. (2003). Dificuldade de aprendizagem: Avaliação do desempenho em provas acadêmicas e cognitivas. Em S. R. F. Enumo \& A. Garcia (Orgs.), Desenvolvimento humano e aprendizagem: Algumas análises e pesquisas (pp. 59-77). São Paulo: Casa do Psicólogo.

Dube, W. \& McIlvane, W. (1996). Implications of a stimulus of a control topography analysis for emergent behavior and stimulus classes. Em T. R. Zenthal \& P. M. Smeets (Orgs.), Stimulus class formation in humans and animals (pp. 197-218). Amsterdam: Elsevier Science B. V.

Enumo, S. R. F., Lima, M. P., Dias, T. L., Paula, K. M. P., Turini, F. A. \& Gottardi, A. P. (2002). Análise de erros de aritmética de alunos do Ensino Fundamental de Vitória, ES. [Resumo] Em Sociedade Brasileira de Psicologia (Org.), Resumos de Comunicações Científicas, XXXII Reunião Anual de Psicologia (p.10), Florianópolis: SBP.
Galy, E., Camps, J. F. \& Melan, C. (2003). Sequence class formation following learning of short sequences. The Psychological Record, 53, 635-645.

Goldiamond, I. (1962). Perception. Em A. J. Bacharach (Org.), Experimental foundation of clinical psychology (pp. 340-380). New York: Basic Book.

Green, G., Stromer, R. \& Mackay, H. (1993). Relational learning in stimulus sequences. The Psychological Record, 43, 599-616.

Holcomb, W. L., Stromer, R. \& Mackay, H. A. (1997). Transitivity and emergent sequence performance in young children. Journal of the Experimental Analysis of Behavior, 65, 96-124.

Hübner, M. M. \& Marinotti, M. (2002). Crianças com dificuldades escolares. Em E. Silvares (Org.), Estudos de caso em psicologia comportamental infantil (pp.259-304). Campinas: Papirus.

Lazar, R. (1977). Extending sequence-class membership with matching to sample. Journal of the Experimental Analysis of Behavior, 27, 381-392.

Lazar, R. \& Kotlarchyk, B. J. (1986). Second order control of sequence class equivalence in children. Behavioral Processes, 13, 205-215.

Lima, M. P. L. \& Assis, G. J. A. (2003). Emergência de classes sequienciais após treino com pareamento consistente. Psicologia: Teoria e Pesquisa, 19(1), 75-84.

Mackay, H., Kotlarchyk, B. \& Stromer, R. (1997). Stimulus classes, stimulus sequences, and generative behavior. Em D. Baer \& E. M. Pinkston (Orgs.), Environmment and Behavior (pp.124-137). Boulder: Westview Press.

Mackay, H.A., Stromer, R. \& Serna, R.W. (1998). Emergent behavior and intellectual functioning: stimulus classes, generalization, and transfer. Em S. Soraci \& W. J. McIlvane (Orgs.), Perspective on fundamental process in intellectual functioning (pp. 287-310). Norwood: Ablex.

Maydak, M., Stromer, R., Mackay, H. \& Stoddard, L. (1995). Stimulus classes in matching to sample and sequence production: The emergence of numeric relations. Research in Developmental Disabilities, 16, 179-204.

McIlvane, W. J., Serna, R. W. Dube, W. V. \& Stromer, R. (2000). Stimulus control topography coherence and stimulus equivalence: Reconciling test outcomes with theory. Em J. C. Leslie. \& D. Blackman (Orgs.), Experimental and applied analysis of human behavior (pp.85-110). Reno: Context Press.

Nascimento, J. V, Assis, G. J. A. \& Baptista. M. Q. G. (2002). Sistema computadorizado para o ensino de leitura e de relações condicionais e seqüenciais - REL 3.0. Belém: UFPA.

Prado, P. S. T. (2001). Ensinando o conceito de número: Contribuições do paradigma de relações. Tese de Doutorado, Universidade de São Paulo, São Paulo.

Sampaio, M. E. C. \& Assis, G. J. A. (2005). Equivalência de estímulos seqüenciais em portadores de necessidades educacionais especiais. Acta Comportamentalia, 13(2), 111143.

Sidman, M. \& Tailby, W. (1982). Conditional discrimination versus matching-to-sample: An expansion of the testing paradigm. Journal of the Experimental Analysis of Behavior, 37, 5-22.

Sidman, M. (1986). Functional analysis of emergent verbal classes. Em T. Thompson \& M. D. L. Zeiler (Orgs.), Analysis and integration of behavioral units (pp. 213-245). New. Jersey: Lawrence Erlbaum Associates Publishers. 
Sidman, M. (1994). Equivalence relations and behavior: A research story. Boston: Authors Cooperative, Inc. Publishers.

Sigurdardottir, Z., Green, G. \& Saunders, R. (1990). Equivalence classes generated by sequence training. Journal of the Experimental Analysis of Behavior, 53, 47-63.

Skinner, B. F. (1972). Tecnologia de ensino (R. Azzi, Trad.). São Paulo: EPU. (Trabalho original publicado em 1968)

Skinner, B. F. (1992). Verbal behavior. Acton, Massachusetts: Copley Publishing Group. (Trabalho original publicado em 1957)

Stevens, S. S. (1951). Mathematics, measurement and psychophysics. Em S. S. Stevens (Org.), Handbook of experimental psychology (pp.1-49). New York: John Wiley \& Sons, Inc.
Stromer, R. \& Mackay, H. A. (1990). A note on the study of transitive relation in stimulus sequence. Experimental Analysis of Human Behavior Bulletin, 8, 2-5.

Stromer, R. \& Mackay, H. A. (1993). Human sequential behavior: Relations among stimuli, class formation, and derived sequences. The Psychological Record, 43, 107-131.

Whaley, D. L. \& Malott, R. W. (1981). Princípios elementares do comportamento (M. M. Matos, M. L. Ferrara \& C. F. Santoro, Trads.). São Paulo: EPU. (Trabalho originalmente publicado em 1971)

Recebido em 19.07.2005

Primeira decisão editorial em 28.03.2006

Versão final em 16.11.2006

Aceito em 26.01.2007 